\title{
A Rare Side Effect Secondary to Warfarin Use: Retropharyngeal Hematoma
}

Burcu YILMAZ1', Hatice Şeyma AKÇA1', Gökhan Aksel'1, Serdar ÖZDEMIR'1, Abdullah ALGIN1', Serkan Emre EROĞLU1

'University of Health Sciences, Ümraniye Education and Research Hospital, Department of Emergency Medicine, Istanbul, Turkey.

\begin{abstract}
Introduction: In this case report, we aimed to explain the importance of urgent management in the diagnosis and treatment of a patient who developed retropharyngeal hematoma as a result of the toxicity of warfarin he used due to atrial fibrillation.

Case Report: A 76-year-old male patient came to the emergency room with complaints of sore throat, difficulty swallowing, and shortness of breath. At the time of admission of the patient using warfarin due to atrial fibrillation, oropharynx examination revealed normal uvula, hematoma near the right lip mucosa, and stridor was detected in the lung auscultation of the patient whose trachea was deviated to the left. In the laboratory tests at the time of the patient's admission, INR was: 13.4. The patient was given $10 \mathrm{mg}$ phytomenadione(K1) iv infusion and $80 \mathrm{mg}$ prednisolone iv. 4 units(1000 ml) of FFP(fresh frozen plasma) was requested. The patient who developed respiratory distress was intubated and transferred to intensive care unit.
\end{abstract}

Conclusion: Warfarin toxicity may rarely cause bleeding in the upper respiratory tract, and if it creates pressure on the respiratory tract, it may pose a serious life-threatening risk.

Keywords: Warfarin, hematoma, retrofarengeal hematoma

\section{Introduction}

Bleeding into the upper respiratory tract secondary to anticoagulant use is a rare but life-threatening condition. It is vital that the pathology be recognized and treatment started as soon as possible.

In this presentation, we aimed to explain the toxicity of warfarin, which he used for atrial fibrillation, and the importance of emergency management in retropharyngeal hematoma examination, diagnosis and treatment.

\section{Case Report}

A 76-year-old male patient came to the emergency room with complaints of sore throat, difficulty swallowing, and shortness of breath for 4 days. At the time of admission of the patient with known chronic renal failure who used warfarin due to atrial fibrillation, the pulse rate was 100/ min, blood pressure:124/79 mmHg, Spo2 was 96\%. Physical examination revealed a glaskow coma scale 15, palpable swelling and hematoma on the right neck (Figure.1). Oropharynx examination revealed normal uvula, hematoma near the right lip mucosa, and stridor was detected in the lung auscultation of the patient whose trachea was deviated to the left (Figure 2).
In the laboratory examinations at the time of the patient's admission, creatine: $2.16 \mathrm{~mm} / \mathrm{dl}$, BUN (blood urea nitrogen):117 mg/dL, hemoglobin:9.9 g/dL, platelet: 295,000/ $\mathrm{uL}$, INR: 13.4. There was no pathology in other laboratory findings

The patient was given $10 \mathrm{mg}$ phytomenadione $(\mathrm{K} 1)$ iv infusion and $80 \mathrm{mg}$ prednisolone iv. 4 units $(1000 \mathrm{ml})$ of FFP (compensated plasma) was requested, intubation decision was made for the patient whose respiratory distress worsened. Cardiopulmonary resuscitation was performed according to AHA criteria for 5 minutes in the patient who developed cardiac arrest secondary to respiratory arrest before being intubated. The patient who met difficult intubation criteria due to retropharyngeal hematoma was intubated with a bougie.

$1000 \mathrm{mg}$ TDP was applied. The patient was transferred to intensive care unit. Inintensivecare, tranexamicacid 250 $\mathrm{mg}$ iv and $10 \mathrm{mg}$ phytomenadione iv infusion were administered for 3 days. The patient, whose neck hematoma completely healed, was extubated 7 days later, and was discharged 15 days later in good health.

\section{Discussion}

Oral anticoagulants are frequently used in patients with a history of atrial fibrillation, prosthetic valve disease, myo- 


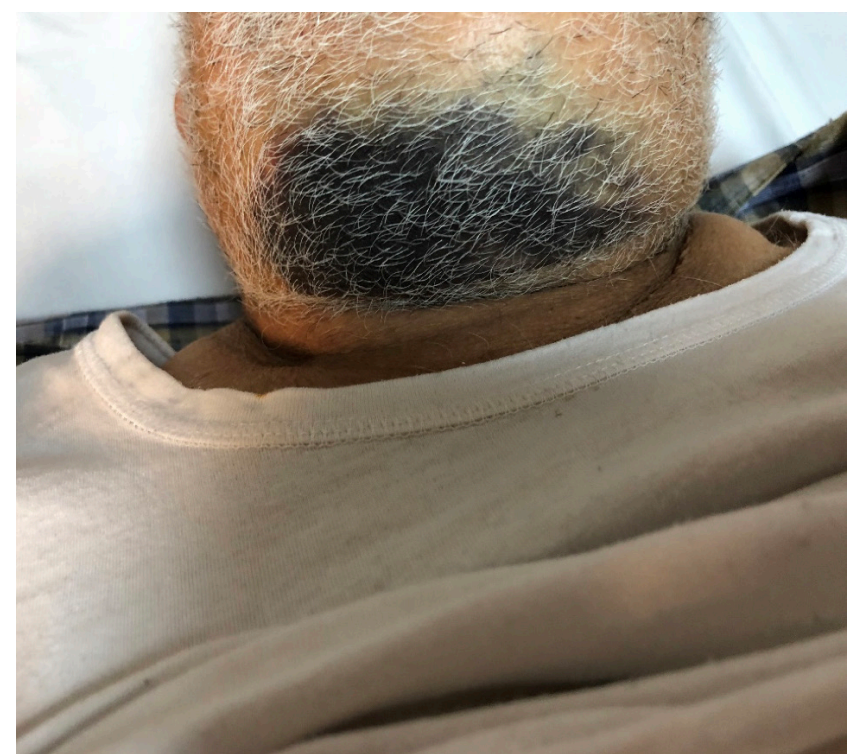

Figure 1: Patient with hematoma on the right neck

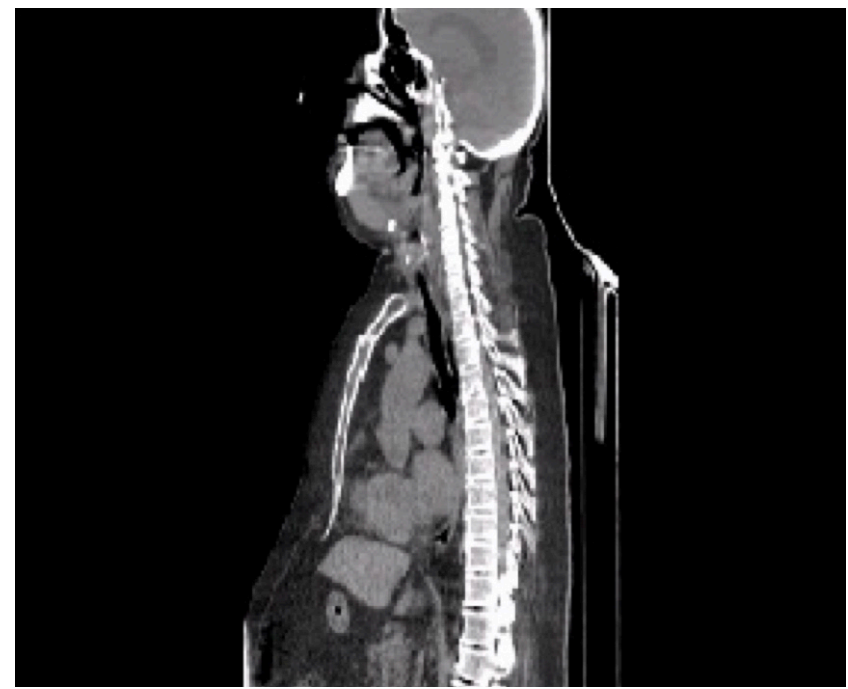

Figure 2: $\mathrm{CT}$ of the patient

cardial infarction, arterial or venous thromboembolism. The efficacy of warfarin is measured by INR, and the use of warfarin is difficult due to genetic differences in dose response, narrow therapeutic range of warfarin, and drug-diet interaction. The risk of haemorrhage secondary to anticoagulant use is most often in the gastrointestinal system, genitourinary system, nose, skin, and retroperitoneum ${ }^{1}$.

Warfarin inhibits vitamin $\mathrm{K}$ reductase and cycloepoxitreductase, which are involved in the carboxylation of factor 2,7,9,10 and other vitamin K-related proteins, preventing the activation of coagulation factors and inhibiting coagulation ${ }^{2}$.

In patients who need to use warfarin, discontinuation of the drug may cause clinical symptoms such as arterial occlusion $^{3}$. In a study comparing new generation anticoagulants, two groups using Dabigatran and Rivoraxaban were compared, and these groups were not superior to each other in terms of complication rate 4 .
Hematomas causing obstruction in the upper respiratory tract are rare, and patients may present with dyspnea, styrodor, dysphagia, odynophagia, neck swelling, and pain in the throat ${ }^{1,2,5}$. Bleeding may be due to trauma, vigorous tooth brushing, local infection, severe coughing, sneezing, yelling, or it may be spontaneous ${ }^{1}$. 'Capps triad', which includes evidence of ecchymosis on the anterior face of the neck and chest, tracheal displacement, and evidence of tracheal and / or esophageal compression ${ }^{5}$. The etiology was also unremarkable, he had dyspnea, stridor, dysphagia, odynophagia and sore throat, and he was in line with the Capps tradition.

The mainstay of retropharyngeal hematoma treatment should be to control coagulability and protect the respiratory tract. Intubation, tracheostomy and cricotrioromia may be considered depending on the clinical situation. Corticosteroids may be given to resolve edema ${ }^{1}$. Tomography or magnetic resonance may be ordered to better visualize the hematoma. The conservative method may take 3 weeks for the symptom and underlying hematoma to resolve $e^{5}$. Our patient was urgently decided to intubate, and he could only be intubated with a bougie. Meanwhile, cardiopulmonary resuscitation was applied to the patient, who developed cardiac arrest, for 5 minutes, and his return to sinus rhythm was ensured by preventing him to remain hypoxic.

Patients with INR $>9$ and no significant bleeding can be given vitamin $\mathrm{K} 12.5-5 \mathrm{mg}$, significantly decreasing INR within 24-48 hours 1 . K1 administration is not recommended unless there is INR $>10$ or bleeding occurs. If toxic levels of intake are considered for suicide, activated charcoal can be given within 1 hour. If there is a life-threatening condition due to warfarin toxicity coagulation, the patient; Factor 4 or 3 prothrombin complex concentrate or fresh frozen plasma and intravenous Vitamin K1 $10 \mathrm{mg}$ should be administered, $\mathrm{K} 1$ can be administered every 12 hours as needed. Tranexamic acid can be considered ${ }^{6}$.

We applied vitamin $\mathrm{K} 1$ and fresh frozen plasma to our patient with INR: 13.4. In previous studies, retropharyngeal hematoma cases were reported due to trauma, surgical complications, clopidogrel use, or warfarin intoxication as in our study $^{6-8}$. Although higher levels are reported in the literature, we think that our high INR level will contribute to the literature.

\section{Conclusion}

Warfarin toxicity may rarely cause bleeding in the upper respiratory tract, and if it creates pressure on the respiratory tract, it may pose a serious life-threatening risk. Detailed anamnesis and physical examination of patients with classical upper respiratory tract infection history such as sore throat and difficulty swallowing can be life-saving. 


\section{References}

1. Y1. Yaman H, Guven D, Kandis H, Subasi B, Alkan N, Yilmaz S. Sublingual and Supraglottic Haemorrhage as a Complication of Warfarin Therapy. Hong Kong J Emerg Med. 2011;18(3):177-181. Doi:10.1177/102490791101800310

2. Toker I, Duman atilla O, Yesilaras M, Ursavas B. Retropharyngeal Hematoma due to Oral Warfarin Usage. Turk J Emerg Med. 2014;14(4):182-184. doi:10.5505/1304.7361.2014.2559 4.

3. Akça HS, Algın A, Yönak H, Eroğlu SE. Arterial occlusion after cessation of warfarin treatment. Cukurova Med J 2020;45(1):381-2. Doi:10.17826/cumj.650126.

4. Aydın İ, Poyraz MK, Algın A, Aksel G, Eroğlu SE. Comparison of Clinical and Biochemical Parameters in Atrial Fibrillation Patients Using Dabigatran and Rivoraxaban and Their Rela- tionship with Complications. İstanbul Med J 2020;21(2):1205. Doi: 10.4274/imj.galenos.2020.32650.

4. Betten DP, Jaquint JL. Traumatic Retropharyngeal Hematoma in a Patient Taking Clopidogrel. Case Rep Emerg Med. 2018;2018: 6147473. Doi:10.1155/2018/6147473

5. Deaton JG, Nappe TM. Warfarin Toxicity. In: Stat Pearls. Stat Pearls August 31, 2020. http://www.ncbi.nlm.nih.gov/books/ NBK431112/

6. Park JE, Jeong EK, Kang DH, Jeon SR. Surgical Treatment of a Life-Threatening Large Retropharyngeal Hematoma after Minor Trauma: Two Case Reports and a Literature Review. Journal of Korean Neurosurgical Society. 2015;58(3):304-7. Doi: 10.3340/jkns.2015.58.3.304.

7. Akoğlu E, Seyfeli E, Akoğlu S, Karazincir S, Okuyucu S, Dağlı AS. Retropharyngeal hematoma as a complication of anticoagulation therapy. Ear, Nose, \& Throat Journal, 2008;87(3):156-9. 